\title{
A Compton camera prototype for prompt gamma medical imaging
}

\author{
P.G. Thirolf ${ }^{1}$, S. Aldawood ${ }^{1,2}$, M. Böhmer ${ }^{3}$, J. Bortfeldt ${ }^{1}$, \\ I. Castelhano ${ }^{1,4}$, G. Dedes ${ }^{1}$, F. Fiedler ${ }^{6}$, R. Gernhäuser ${ }^{3}$, \\ C. Golnik ${ }^{5}$, S. Helmbrecht ${ }^{6}$, F. Hueso-GonzÁlez ${ }^{6}$, H. V.D.

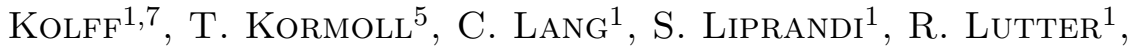 \\ T. Marinšek ${ }^{1}$, L. Maier ${ }^{3}$, G. Pausch ${ }^{5}$, J. Petzoldt ${ }^{5}$, K. RÖMer ${ }^{6}$, \\ D. $\mathrm{SChAART}^{7}$ and K. PAROdi ${ }^{1}$ \\ ${ }^{1}$ Ludwig-Maximilians-Universität München, 85748 Garching, Germany \\ ${ }^{2}$ King Saud University, Riyadh, Saudi Arabia, \\ ${ }^{3}$ Technische Universität München, 85748 Garching, Germany \\ ${ }^{4}$ University of Lisbon, Lisbon, Portugal \\ ${ }^{5}$ OncoRay - National Center for Radiation Research in Oncology and \\ Technische Universität Dresden, Germany \\ ${ }^{6}$ Helmholtz-Zentrum Dresden Rossendorf, Dresden, Germany \\ ${ }^{7}$ Delft University of Technology, Delft, The Netherlands
}

\begin{abstract}
A Compton camera prototype for a position-sensitive detection of prompt $\gamma$ rays from proton-induced nuclear reactions is being developed in Garching. The detector system allows to track the Comptonscattered electrons. The camera consists of a monolithic $\mathrm{LaBr}_{3}$ :Ce scintillation absorber crystal, read out by a multi-anode PMT, preceded by a stacked array of 6 double-sided silicon strip detectors acting as scatterers. The $\mathrm{LaBr}_{3}$ :Ce crystal has been characterized with radioactive sources. Online commissioning measurements were performed with a pulsed deuteron beam at the Garching Tandem accelerator and with a clinical proton beam at the OncoRay facility in Dresden. The determination of the interaction point of the photons in the monolithic crystal was investigated.
\end{abstract}




\section{Introduction}

In order to be able to fully exploit the beneficial properties of tumour treatment by particle beams (protons, ions), provided by the highly conformal dose deposition within the Bragg peak, precise knowledge of the ion beam stopping range is mandatory. Our approach towards this goal is to detect the prompt photon range in tissue emitted during nuclear interactions between the hadron beam and the organic target as surrogate signal of the stopping range. The Compton scattering process can be exploited to reconstruct the $\gamma$ incidence direction from the Compton scattering kinematics of the primary photon. Perspectives of prompt- $\gamma$ imaging have been extensively studied both theoretically and experimentally [1-4], and Compton camera systems have been studied by several groups [5-10]. Fig. 1 displays the principle of a Compton camera, consisting of a scatterer and absorber component: in ' $\gamma$-tracking mode' (left panel), the position dependent detection of the scattering and absorption interaction of the impinging $\gamma$ ray (with full absorption of the photon energy in the absorber) allows to reconstruct the photon source position on the surface of the 'Compton cone'. Intersecting reconstructed Compton cones from various events finally reveals the source position, as visible from the bottom row of Fig. 1, where this is shown for one $\left(\mathrm{N}_{\gamma}=1\right)$ and 10 events $\left(\mathrm{N}_{\gamma}=10\right)$, respectively $[9,10]$. In case

a)

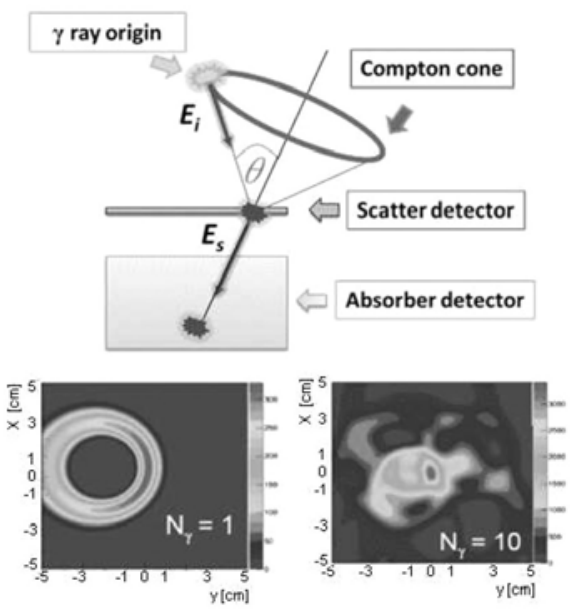

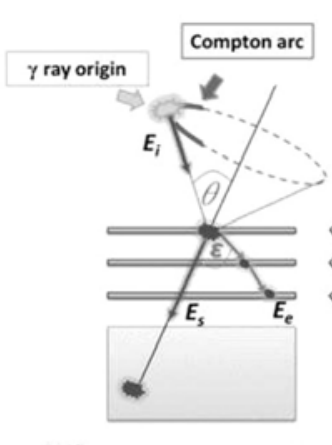

b)

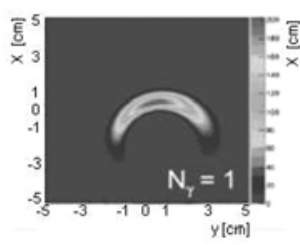

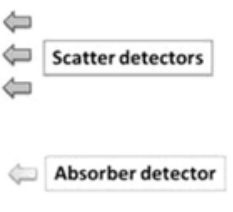

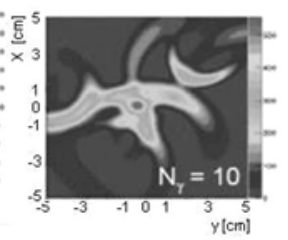

Figure 1: Principle of a Compton camera: a) with $\gamma$ tracking only, b) with additional electron tracking capability. 
of sufficiently high photon energies (few $\mathrm{MeV}$ ), a layered structure of the scatterer detector (e.g. by an array of position-sensitive silicon strip detectors) enables a tracking of the Compton electron. This not only reduces the Compton cone to an arc segment (see bottom row), but allows to include also incompletely absorbed events into the source reconstruction, thus increasing the reconstruction efficiency $[10,11]$.

\section{Compton camera layout}

The Compton camera prototype, presently being under commissioning in Garching, is composed of a scatterer/tracker array consisting of 6 doublesided silicon strip detectors (DSSSD, area: $50 \times 50 \mathrm{~mm}^{2}$, thickness: $0.5 \mathrm{~mm}$, 128 strips/side) and a monolithic $\mathrm{LaBr}_{3}$ :Ce scintillation absorber crystal $\left(50 \times 50 \times 30 \mathrm{~mm}^{3}\right)$, read out by a multi-anode PMT (Hamamatsu H9500, $16 \times 16$ segments with $3 \times 3 \mathrm{~mm}^{2}$ pixel size). The strip detectors are read out
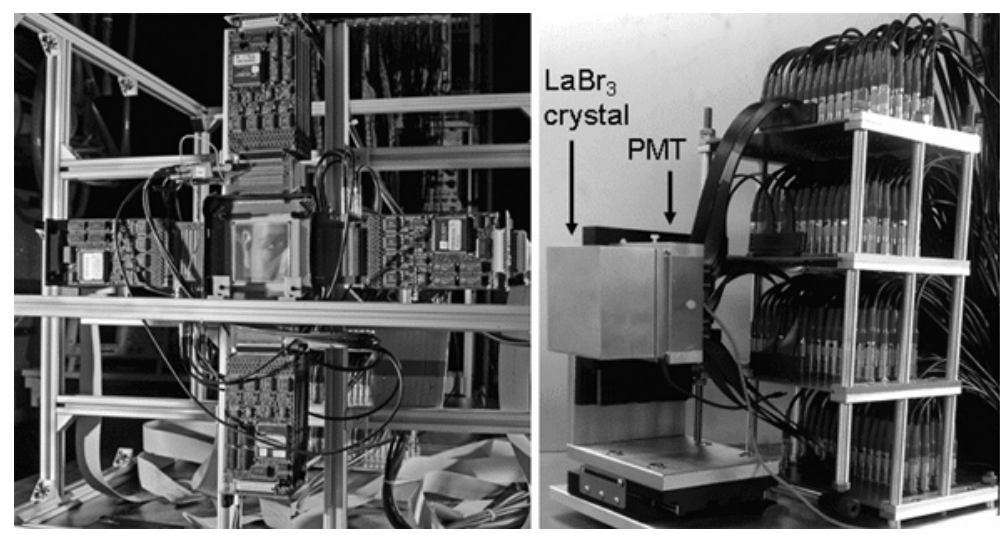

Figure 2: Left: Front of the Compton camera scatter/tracker array: double-sided Si-strip detector with ASIC-based signal processing electronics. Right: Absorbing $\mathrm{LaBr}_{3}$ :Ce scintillator.

via a compact ASIC-based electronics [12], while the signals from PMT segments of the absorbing scintillator are processed via individual channels of spectroscopy electronics. Energy signals are digitized in VME-based chargeto-digital converters (QDC, mesytec MQDC-32), while timing signals can be acquired via time-to-digital converters (mesytec MTDC-32). Fig. 2 shows the front sight of the scatterer array with its surrounding, directly attached signal processing boards (left), while the $\mathrm{LaBr}_{3}$ :Ce scintillator with its 256 signal outputs can be seen on the right-hand side. Design simulation studies 

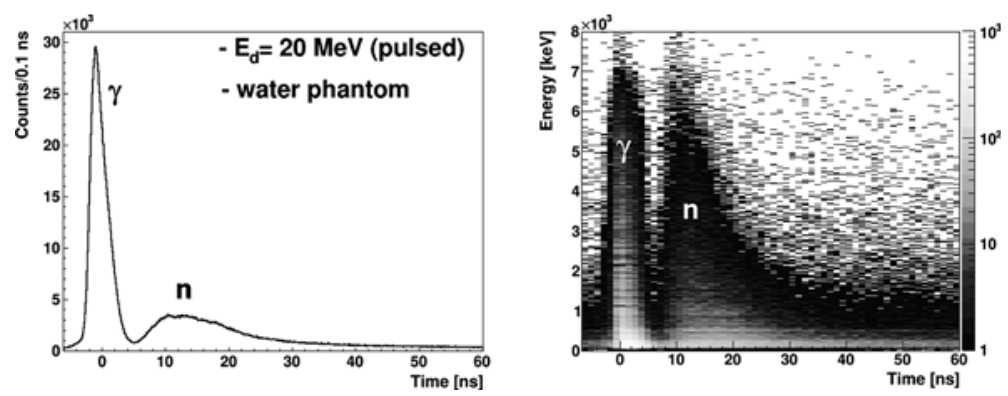

Figure 3: Time spectrum acquired with the $\mathrm{LaBr}_{3}$ :Ce scintillator of the Compton camera from a pulsed deuteron beam $\left(\mathrm{E}_{d}=20 \mathrm{MeV}\right)$ hitting a water phantom. Right panel: photon detection time (relative to RF trigger signal) vs. photon energy, left panel: projection on time axis.

with GEANT4 predict an angular resolution of $\theta \approx 2^{\circ}$ and an image reconstruction efficiency of $10^{-3}-10^{-5}\left(\mathrm{E}_{\gamma}=2-6 \mathrm{MeV}\right)$ for a camera arragment with $50 \mathrm{~mm}$ distance between the first scatterer detector and the photon source (representing a small animal irradiation scenario) [10,11].

\section{Characterization of camera components}

The $\mathrm{LaBr}_{3}$ :Ce detector crystal (with reflective side surface coating) has been characterized in the laboratory with calibration sources. Using a collimated $2 \mathrm{D}$ irradiation of the front surface with $662 \mathrm{keV}$ from ${ }^{137} \mathrm{Cs}$, an average relative energy resolution of $\Delta E_{\gamma} / E_{\gamma}=3.8 \%$ (FWHM) was obtained, independent of the irradiation position. Relative to a fast plastic (BC-418) reference detector, a time resolution of 270 ps was obtained for the coincidence of photons from ${ }^{60} \mathrm{Co}$.

Using a $20 \mathrm{MeV}$ pulsed deuteron beam (pulse distance $200 \mathrm{~ns}$, pulse width $6 \mathrm{~ns}$ ) from the Garching Tandem accelerator hitting a water phantom, the time spectrum (relative to the RF trigger signal) shown in Fig. 3 was acquired with the scintillator, showing the well-separated prompt photon and delayed neutron component (right: $\gamma$ arrival time vs. photon energy, left: projection on time axis).

Using the clinical proton beam of the research area at the Universitäts Protonen Therapie Dresden (UPTD), water and polymethyl methacrylate (PMMA) phantoms, respectively, were bombarded with 100, 160 and 225 $\mathrm{MeV}$ protons (pulse distance $9.4 \mathrm{~ns}$, according to the cyclotron frequency of $106 \mathrm{MHz}$ ). Fig. 4 displays the resulting time spectrum versus the photon energy for $\mathrm{E}_{p}=225 \mathrm{MeV}$ hitting the water phantom, indicating the pulse 


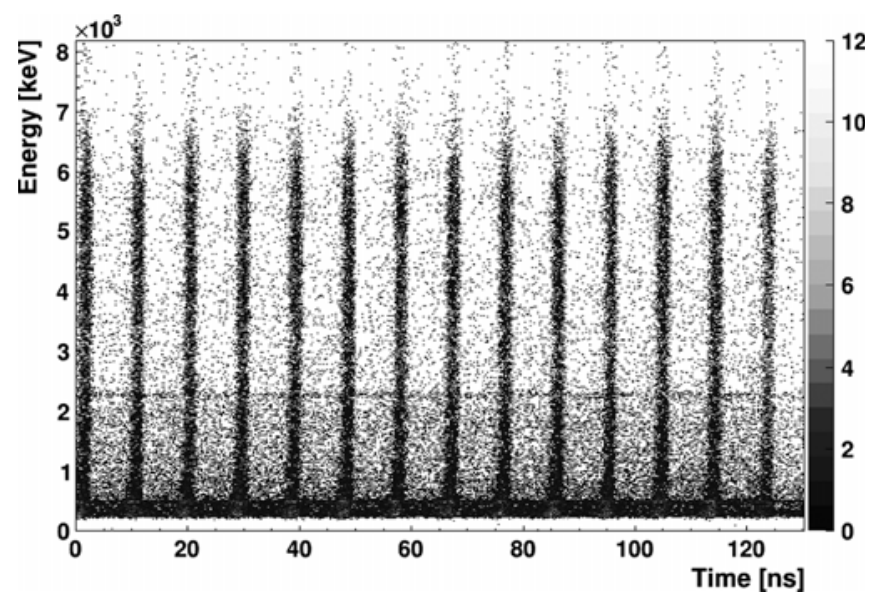

Figure 4: Timing spectrum for $225 \mathrm{MeV}$ protons hitting a water phantom with a pulse period of $9.4 \mathrm{~ns}$, as measured with the $\mathrm{LaBr}_{3}$ :Ce scintillator of the Compton camera.

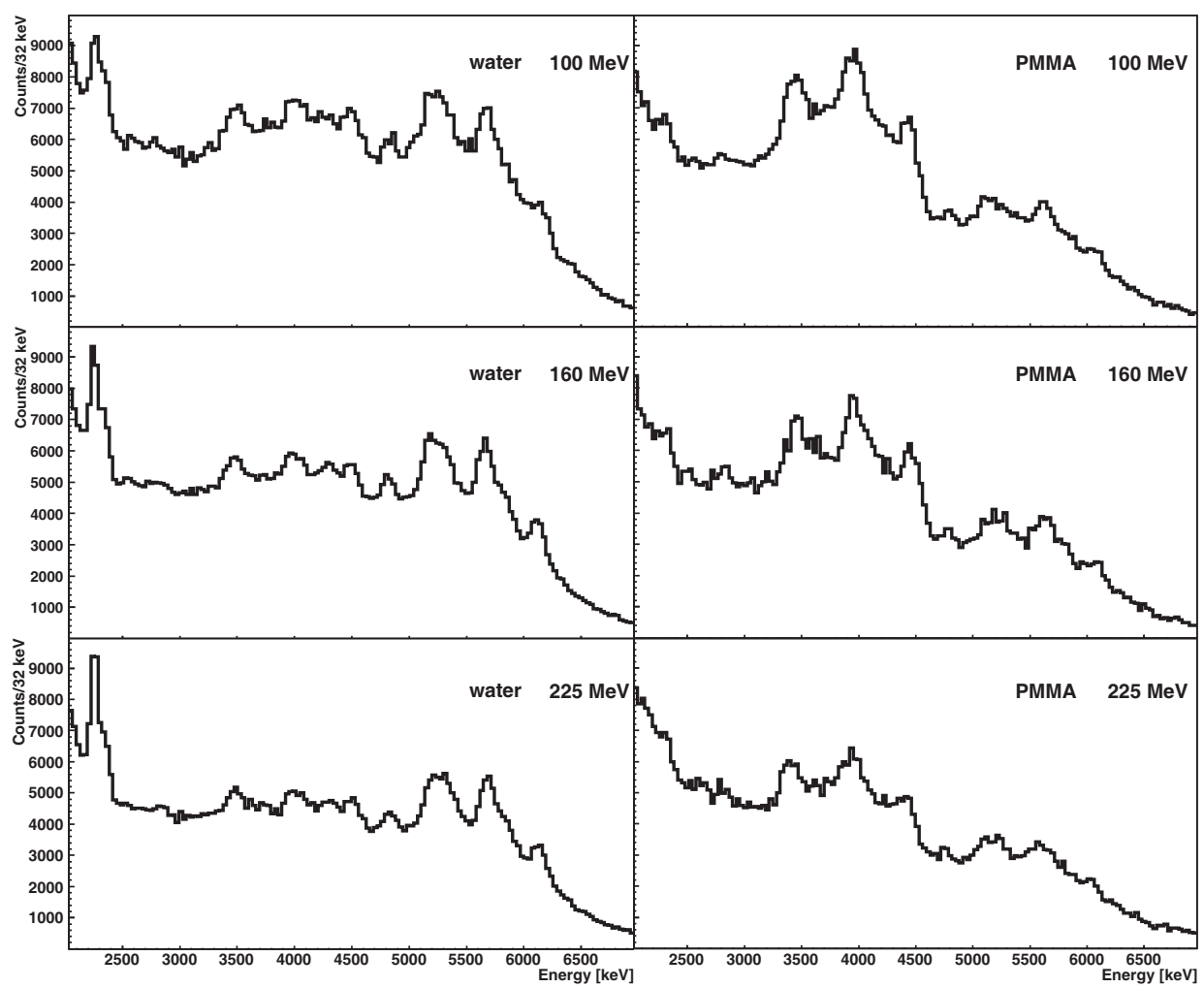

Figure 5: Prompt photon energy spectra from 100, 160, $225 \mathrm{MeV}$ protons hitting a water (left column) and a PMMA phantom (right column). 


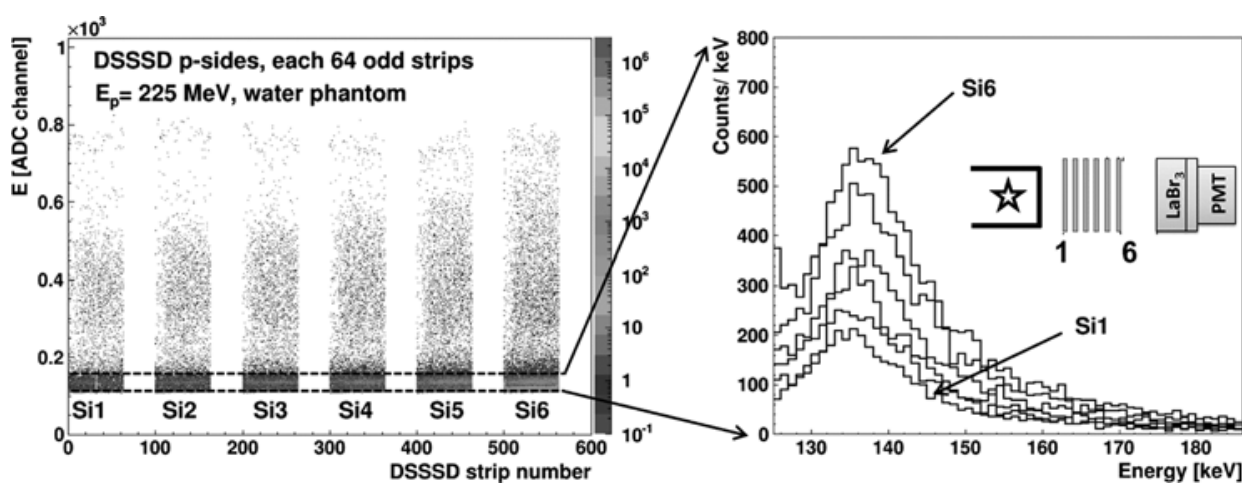

Figure 6: Energy loss spectra measured with the DSSSD array for $225 \mathrm{MeV}$ protons and the water phantom. Exemplarily shown are the digitized energies from the odd 64 p-side strips of detector 1 (close to the phantom) to detector 6 (in front of the scintillator). The zoomed region displayed in the right part highlights the energy deposit by Compton scattered electrons successively accumulating in intensity across the scatterer array towards the scintillator.

structure. The horizontal line at $2.2 \mathrm{MeV}$ represents the capture of a neutron, releasing the deuteron binding energy. Obviously, no strong neutron background component is visible.

The prompt photon energy spectra measured with the $\mathrm{LaBr}_{3}$ :Ce scintillator both for the water (left column) and PMMA phantom (right column) are shown in Fig. 5 for the three beam energies of 100, 160 and $225 \mathrm{MeV}$, respectively. The energy spectra from the water phantom show as most prominent feature the deexcitation from the first excited state of ${ }^{16} \mathrm{O}$ at 6.13 $\mathrm{MeV}$ plus the corresponding single- and double-escape peaks, while for the PMMA phantom the contribution from excited ${ }^{12} \mathrm{C}(4.44 \mathrm{MeV}$ and its escape peaks) can be observed much stronger compared to the water phantom.

Data from part of the scatterer array (for $225 \mathrm{MeV}$ protons hitting the water phantom) are shown in Fig. 6 (left part). Energy loss signals are displayed exemplarily for the 64 odd strips of the p sides of the 6 DSSSDs 'Si 1' to 'Si 6'. The structure around $130 \mathrm{keV}$ reflects the energy deposition of Compton scattered electrons as a zoomed projection of the spectra shown on the right side onto the energy axis. The intensity contributed by Compton electron successively accumulates along the path of primary photons through the DSSSD stack, therefore exhibiting increasing intensity with increasing proximity to the absorbing scintillator placed behind DSSSD 'Si 6'.

A mandatory prerequisite for photon source reconstruction using the Compton camera is the determination of the primary interaction position 


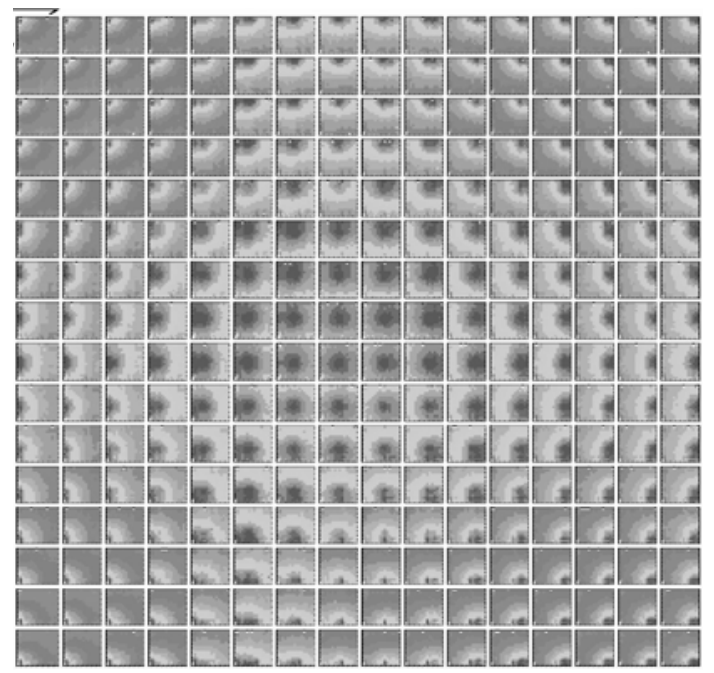

Figure 7: Grid scan of $16 \times 16$ irradiation positions of the monolithic $\mathrm{LaBr}_{3}: \mathrm{Ce}$ detector, using a $1 \mathrm{~mm}$ collimated ${ }^{137} \mathrm{Cs}$ source and a grid step size of $3 \mathrm{~mm}$ in $\mathrm{x}$ and y direction. All 256 segments of the multi-anode PMT are individually read out. The resulting $2 \mathrm{D}(16 \times 16)$ light amplitude distribution of each irradiation position clearly indicates the source position movement from the upper left corner to the lower right corner.

of photons hitting the absorbing scintillator. The 'k-Nearest Neighbour' algorithm, developed at TU Delft [13], is used to derive spatial information from the monolithic scintillator. It is based on a reference library of $2 \mathrm{D}$ light amplitude distributions, obtained from a 2D scan of the detector front surface with a narrowly collimated calibration source. In Fig. 7, we show the resulting $2 \mathrm{D}$ light amplitude map obtained from a grid scan with $16 \times 16$ irradiations positions (step size $3 \mathrm{~mm}$ in 2 dimensions), using a $1 \mathrm{~mm}$ collimated ${ }^{137} \mathrm{Cs}$ source $(82 \mathrm{MBq})$. The correlation of the displayed 256 light amplitude distributions with their respective irradiation position, continuously moving from the upper left corner of the crystal to the lower right corner, is clearly visible. A similar scan with $102 \times 102$ irradiation positions (0.5 mm grid step size, 20 seconds measurement time per position) was performed to create the reference library needed for the k-NN algorithm. In order to derive the spatial resolution, irradiation position coordinates of $\mathrm{k}=$ 2000 closest matching reference light amplitude distributions, derived from a calculation of the Euclidean distance of the distribution of an 'unknown' event to every distribution from the reference set (with 400 events analyzed for each of the ca. $10^{4}$ irradiation positions), were accumulated into a $2 \mathrm{D}$ 

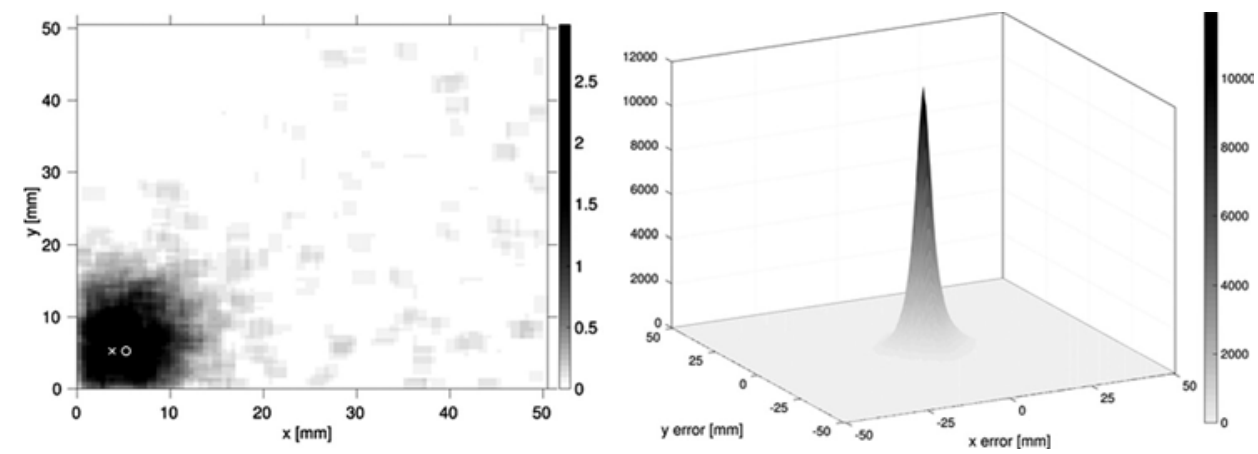

Figure 8: Left: Smoothed histogram of 2000 closest matching 2D light distributions for each 'unknown' irradiation event selected by the k-NN algorithm. The cross (open circle) denotes the actual (calculated) irradiation position. Right: Error histogram of the k-NN algorithm containing the distances between actual and calculated irradiation position for each of the events in the reference library. The corresponding FWHM indicates the spatial resolution.

histogram. After smoothing, the maximum of this distribution (displayed in the left part of Fig. 8) was identified with the calculated source position.

The spatial resolution can be quantified by performing this procedure for each of the reference events, treated as 'unknown'. The differences between actual and reconstructed source position are filled into an 'error histogram' (see right panel of Fig. 8) and the FWHM of this distribution reflects the corresponding spatial resolution. So far, a spatial resolution of 5.5(5) $\mathrm{mm}$ has been achieved, with room for further improvement, e.g., by using a tighter collimator $(0.5 \mathrm{~mm})$.

In conclusion, the detector components of a Compton camera, designed for prompt- $\gamma$ ray based imaging of the particle beam range in hadron therapy and consisting of a stack of 6 doubled-sided Si strip detectors as scatterers (allowing also for Compton electron tracking) and a $\mathrm{LaBr}_{3}$ :Ce scintillator, have been successfully commissioned off- and online. All prerequisites have been achieved that enable to study the photon source reconstruction capabilities of the Compton camera prototype as the next step on the road towards an online ion beam range verification device.

This work is supported by the DFG Cluster of Excellence MAP (MunichCentre for Advanced Photonics). 


\section{References}

[1] C.H. Min et al., Applied Phys. Lett. 89, 183517 (2006).

[2] E. Testa et al., Appl. Phys. Lett. 93, 093506 (2008).

[3] F. Fiedler et al., Nucl. Sci. Symp. Conf. Rec. (NSS/MIC), 2011 IEEE.

[4] J.C. Polf et al., Phys. Med. Biol. 59, 2325 (2014).

[5] F. Roellinghoff et al., Nucl. Instr. Meth. A648(2011) S20.

[6] X. Lojacono et al., Nucl. Sci. Symp. Conf. Rec., 2011 IEEE 1, 3518.

[7] T. Kormoll et al., Nucl. Instr. Meth. A626 (2011) 114.

[8] M.-H. Richard et al., IEEE Trans. Nucl. Sci. 58 (2011).

[9] P.G. Thirolf et al., EPJ Web of Conferences 66 (2014) 11036.

[10] C. Lang et al., Journal of Instrumentation 9 (2014) P01008.

[11] C. Lang, PhD thesis, LMU Munich (2015).

[12] W. Beusch et al., Internal Note, CERN-ECP/94-17 (1994).

[13] H.T. van Dam et al., IEEE Trans. Nucl. Sci. 58 (2011) 2139. 